Myeloid sarcoma (MS) is a malignant extramedullary tumour, which consists of immature cells of myeloid origin. It may occur de novo, concurrently or precede the diagnosis of acute myeloid leukemia (AML), myelodysplastic syndrome (MDS) or chronic myeloid leukemia (CML). MS can also be a manifestation of the relapse of the disease. The more frequent sites of involvement are the skin, orbit, bone, periosteum, lymph nodes, gastrointestinal tract, soft tissue, central nervous system and testis. Because of its different localization and symptoms, and the lack of diagnostics algorithm, myeloid sarcoma is a real diagnostic challenge, in particular in patients without initial bone marrow involvement. The correct diagnosis of MS is important for adequate therapy, which is often delayed because of a high misdiagnosis rate. In the paper, the role of immunohistochemistry, cytogenetic and molecular genetic analyses is emphasized as well as the breadth of unclear aspects of this disorder in children.

Key words: myeloid sarcoma, children, diagnosis, therapy.

Contemp Oncol (Pozn) 2016; 20 (6): 444-448 DOI: https://doi.org/10.5114/wo.2016.65602

\section{Myeloid sarcoma in children - diagnostic and therapeutic difficulties}

\author{
Magdalena Samborska', Katarzyna Derwich ${ }^{1}$, Jolanta Skalska-Sadowska', \\ Paweł Kurzawa ${ }^{2}$, Jacek Wachowiak ${ }^{1}$
}

${ }^{1}$ Department of Pediatric Oncology, Hematology and Transplantology, Poznan University of Medical Sciences, Poznan, Poland

${ }^{2}$ Division of Pathomorphology, Karol Jonscher University Hospital, Poznan, Poland

\section{Introduction}

Myeloid sarcoma (MS) is an extramedullary mass, which consists of myeloid blasts with or without maturation, forming a tumour in which the tissue architecture is effaced [1]. It may occur de novo or concurrently with acute myeloid leukemia (AML), myelodysplastic syndrome (MDS) or a myeloproliferative disorder. Myeloid sarcoma can also be a manifestation of the relapse of the disease [2-4]. Non-effacing tissue leukemic infiltrates are distinguished from MS [1]. Myeloid sarcoma was first described in 1853 and termed chloroma because of the greenish color, caused by the presence of myeloperoxidase. The terms granulocytic sarcoma, extramedullary myeloid cell tumour or myeloblastoma have also been used [3, 5].

In adults with AML, the incidence rate of MS is about 2-5\%. In children, it varies in the literature, but is apparently significantly higher as it reaches $40 \%$ in patients with $\mathrm{AML}$ diagnosis $[2,6]$.

The diagnosis of the two entities in the myeloid sarcoma spectrum may be distinctively challenging: de novo MS and isolated MS as AML relapse after allogeneic haematopoetic stem cell transplantation (alloHSCT). The lack of current bone marrow involvement defines both of these conditions.

\section{Pathogenesis}

The mechanism responsible for the accumulation of blasts in extramedullary tissue is not fully understood. One of the reasons for the development of MS in extramedullary sites, is the natural organ barriers, which cause poor penetration of drugs and enable blast cells to survive, despite systemic therapy. These places include the central nervous system (CNS) and reproductive organs [3, 6]. However, development of MS must have an underlying molecular basis. In the study by Stefanidakis et al., it was demonstrated that invasion of blast cells is attributed to the role of invadosome, which is a supramolecular complex of matrix metaloproteinase (MMP-9) and leukocyte B integrin. The authors suggest that inhibition of this leukemia invadosome could be a potential treatment target [7]. The role of MMP-9 has also been indicated in the report by Feng et al., as this protein, together with MMP-2, produced by leukemic cells, increase their ability to disrupt the blood-brain barrier [8]. Wang et al. analyzed a highly invasive monocytic leukemia blast line, called SHI-1 cells, and showed their strong expression of matrix metalloproteinase 2 (MMP-2), membrane type 1 MMP (MT1-MMP) and tissue inhibitor of metalloproteinase 2 (TIMP-2), which also leads to increased invasion capacity [9].

It is also hypothesized that a potential reason for migration of blast cells is interactions between chemokines and chemokine receptors, unique for extramedullary manifestation. Faaij et al. analyzed a group of pediatric AML patients with extramedullary skin manifestation. In their report, skin homing of blast cells is explained by increased number of CCR-2 positive AML cells, while their retention 
in the skin and long survival is attributed to other chemokine interactions, including CCR5/CCL3 and CXCR4/CXCL12 [10].

\section{Clinical manifestation of myeloid sarcoma}

\section{Myeloid sarcoma concurrent with leukemia}

Most commonly, MS occurs concurrently or during the course of AML [2]. There is different data about the frequency of extramedullary disease in children at the moment of diagnosis of leukemia. Kobayashi et al. analyzed a group of 240 pediatric cases of AML - the frequency of extramedullary disease at diagnosis was $23.3 \%$, which included patients with MS and CNS leukemia. Patients with extramedullary presentation of the disease, displayed a higher initial white blood cells count (WBC) and were more frequently diagnosed with type M4 and M5 leukemia [11]. In the study by Dusenbery et al., in the group of 1832 pediatric patients with AML, 10.9\% presented extramedullary manifestation at diagnosis [12]. Johnston et al. reported that out of 1459 analyzed pediatric patients with AML, 99 patients presented myeloid sarcoma, which constitutes $6.7 \%$ [13].

\section{De novo myeloid sarcoma}

De novo (isolated) myeloid sarcoma is defined as the absence of leukemia or MDS history and the lack of current bone marrow involvement [3]. In the analysis by Pileri et al., it constituted $27 \%$ of all MS diagnoses [14]. In children, de novo MS is limited to case reports.

Bain et al. described a 4-month infant, who was diagnosed with skin myeloid sarcoma on the basis of a biopsy, which was performed in a dermatology unit. Interestingly, leukemic involvement of bone marrow in this patient appeared a year after the skin biopsy was performed [4].

Rénard et al. presented a case of 5-year-old boy, diagnosed with isolated abdominal MS, which was manifested as a bowel invagination. Firstly, after the biopsy was performed, the diagnosis of non-Hodgkin lymphoma was established, however further immunohistochemical research confirmed extramedullary manifestation of AML M5 [15].

In the literature, we found no cases of MS preceding CML in a pediatric patient. Two cases of adults with $B C R-A B L$ positive isolated myeloid sarcoma have been reported. Kuan et al. presented the case of a 21-year-old- male, with MS located in the left orbit, paranasal sinuses and anterior cranial fossa [16]. Mitchell et al. reported a case of a 73-year-old man, with MS of thoracic localization with a pleural effusion [17]. Interestingly, in both patients, the $B C R-A B L$ fusion gene was demonstrated in the tumour cells, while it was negative in the bone marrow or peripheral blood $[16,17]$.

\section{Myeloid sarcoma as a relapse after allogeneic haematopoetic stem cell transplantation (alloHSCT)}

Extramedullary relapse is an important complication, which is more frequent in post-transplant patients compared with patients treated without alloHSCT. Its incidence is about $7-46 \%$ of all AML relapses [18]. It may occur with or without bone marrow involvement, but isolated extramedullary relapse rarely occurs; in children it is limited to case reports $[4,18]$.
The risk factors, which have been so far reported, include: a history of extramedullary disease, FAB class M4 or M5 AML, advanced disease status at the time of HSCT and high-risk cytogenetics which includes such abnormalities as chromosome $5 q$ and $7 q$ deletions and FLT-3 mutations. Extramedullary relapse has a higher frequency in children, what may be associated with the increased incidence of M4/M5 leukemia and a prior history of extramedullary disease [6, 16-18].

The role of chronic graft versus host disease (GvHD) as another risk factor, is still being discussed. It is hypothesized that an effect of graft-versus leukemia (GvL) derived from chronic graft versus host disease is diminished in sites other than bone marrow. The reason for that may be the decreased concentration of CD8 positive T-cells in others tissues than bone marrow [19-21].

Adequate diagnosis of isolated MS after alloHSCT may be extremely difficult. Due to the impaired immune defence in this group of patients, infection might be suspected at first. Yoo et al. described a case of a pediatric patient in remission from AML, after a second alloHSCT, who on admission presented a right cheek swelling, right side nasal obstruction and discharge from the nose. He underwent antibiotic and antifungal treatment before a biopsy and the immunohistochemistry enabled the correct final diagnosis of isolated MS to be established [18].

\section{Myeloid sarcoma involved sites}

The symptoms of MS depend on the localization of the tumour. The tumour mass can be located in any part of the body other than bone marrow. The most commonly involved sites include: bone, periosteum, skin, orbit, lymph nodes, gastrointestinal tract and central nervous system. In children, skin and orbital localizations are the most often reported $[2,5,6]$.

\section{Cutaneous myeloid sarcoma}

Cutaneous MS (leukemia cutis) is a cutaneous infiltration of leukemia cells, which results in skin lesions. In children skin infiltration is most common extramedullary manifestation. Most often it occurs in AML, but it may also appear in CML or MDS [3, 22]. In AML patients, skin infiltration is most commonly associated with myelomonocytic and monocytic blast differentiation [3, 6, 22, 23]. The overall incidence is about $10-15 \%$ of patients with AML $[6,22]$. In congenital leukemia frequency of leukemia cutis is about $25-30 \%$ of infants [22, 24]. In rare cases cutaneous MS may precede bone marrow involvement, which makes the diagnosis more challenging $[4,22]$.

According to the study by Hurley et al., the most common localizations were the torso, followed by the upper extremities, lower extremities, head and neck [23]. Skin lesions tend to develop in the places of previous or current inflammation. Their features are not specific, as they are usually described as erythematous papules, nodules or plaques of different sizes. For this reason, cutaneous myeloid sarcoma can mimic many different dermatoses, such as viral exanthema, vitiligo, psoriasis, or seborrheic dermatitis. Sweet's syndrome, which includes skin lesions, fever and neutrophilia must also be excluded, as it may accompany malignancies and is most commonly related to AML [4, 25-27]. 


\section{Orbital myeloid sarcoma}

The orbit area is the second commonest localization of myeloid sarcoma in children. Orbital MS is most often associated with AML M2, M4 and M5 subtypes [6].

The most frequent symptom is unilateral exophtalmus, however it can also present as ptosis, lacrimal gland involvement, concjunctival mass, iridic and diffuse uveal involvement or retinal haemorrhages [28, 29]. These clinical features are not specific for MS as they are described in all orbital tumours. Differential diagnosis in children includes such entities as: rhabdomyosarcoma, neuroblastoma metastases and Burkitt's lymphoma. Neuroblastoma and Ewing's sarcoma usually cause more bone destruction [28].

Computer tomography (CT) and magnetic resonance imaging (MRI) are essential not only in revealing the tumour, but also in planning the biopsy and evaluating the response to treatment [30].

\section{Diagnosis of myeloid sarcoma}

In the diagnostic process of MS, the biopsy of the tumour and immunohistochemical staining are most important. If possible, tissue biopsy is preferable to fine needle biopsy [31]. Flow cytometry, cytogenetic and molecular analysis of the tissue are also recommended. Flow cytometry of the peripheral blood and bone marrow is essential, to exclude systemic disease [2, 5, 31]. Among radiological techniques, $\mathrm{CT}$ and MRI imaging enable evaluation of size and location of the tumour and are helpful in distinguishing tumours from other lesions such as haematomas or abscesses. PET-CT can also be considered as it can detect multiple lesions [31]. Stolzel et al. performed PET-CT in 10 patients with extramedullary acute myeloid leukemia. PET-CT scans confirmed extramedullary involvement in already known locations in 9 out of 10 patients; in 6 patients additional extramedullary sites were detected [32]. PET-CT can also be helpful in planning radiotherapy and to evaluate treatment response [3].

\section{Histology and immmunohistochemistry}

Histologically, MS is an extramedullary tumour which consists of immature granulocytes, monocytes or both [33]. It has been subclassified by morphological features and the extent of maturation into immature granulocytic sarcoma, differentiated granulocytic sarcoma, monoblastic sarcoma, monocytic sarcoma and myelomonocytic sarcoma. However, this division is currently less useful, because of the dominant role of immunohistochemistry and cytogenetic studies in the diagnostic approach [2, 5].

To establish a correct diagnosis of MS, an accurate panel of antibodies must be chosen. In the analysis by Pileri et al. 10 out of 25 de novo MS were misdiagnosed as non-Hodgkin lymphomas. Immunohistochemistry was essential in correcting diagnoses in these cases [14].

Most of the authors agree that the minimal panel must include CD43 and lysosyme.

Together with myeloperoxidase (MPO), CD68 and CD163 markers are believed to identify the majority of MS types [2, 4, 33]. In Alexiev analysis MPO and CD117 reflected myeloid differentiation, while CD68 and CD163 were expressed in monocytic cells [33]. However, in other reports, MPO is considered as not specific marker for MS [5].

In differential diagnosis, special attention should be paid to B-cell and T-cell lymphomas, as non Hodgkin-lymphoma is the most frequent misdiagnosis [2, 5, 34, 35]. To exclude these entities CD20, CD79a and CD3 should be added to the antibodies panel. NK-lymphoma must also be excluded, and it is important to note, that MS can be CD56 positive, which has been observed in a few studies. CD56 and CD4 have been linked to cutaneous extramedullary manifestation of AML [35].

Other tumours, which should be considered in differential diagnosis, include: neuroblastoma, Ewing sarcoma, rhabdomyosarcoma, primitive neuroectodermal tumour, melanoma or medulloblastoma, in the case of localization in the central nervous system $[2,5,33,34]$.

\section{Cytogenetic and molecular genetics}

Cytogenetic and molecular examination plays an important role in the diagnostic process of MS. As MS is most commonly associated with AML, genetic abnormalities characteristic for this entity are frequently found. Translocation (8;21) which results in AML1-ETO fusion gene, is especially frequent in MS of orbital localization [6, 7]. Trisomy 8 is more often associated with cutaneous MS, while inversion of chromosome 16 has been more frequently reported in patients with intraabdominal MS $[2,6,14,36]$. In infants with extramedullary involvement, 11q23 is frequently found. The following abnormalities were also reported to be associated with MS: $\mathrm{t}(9 ; 11)$, del (16q), t(8;17), t(8;16) and t(1;11) [14].

In MS accompanying chronic myeloid leukemia, as the form of its blastic transformation, the BCR-ABL fusion gene can be detected not only in peripheral blood or bone marrow, but also in the tissue of the tumour $[16,17]$.

Only a little data about the molecular abnormalities found in MS is available. Some of authors reported increased frequency of the nuclephosmin1 (NPM1) mutation. Falini et al. detected NPM1 mutation in 15\% of 181 analyzed samples. The authors suggest that the antibody against NPM should be added to the diagnostic panel of MS [37].

\section{Prognosis}

Usually, the presence of MS has been linked to a poor prognosis. However, many authors emphasize that the outcome of patients with MS depends on other prognostic factors, such as presence of genetic abnormalities. Introducing treatment including $\mathrm{AML}$-chemotherapy with the possibility of alloHSCT has changed the view of the survival of this group of patients $[5,6]$.

In the analysis by Tsimberidou et al. it was demonstrated, that EFS is longer in patients with non-leukemic MS compared with AML patients [38]. Interesting data is available about the prognosis of pediatric patients with MS. Johnston et al. compared survival of patients with orbital myeloid sarcoma, central nervous system myeloid sarcoma and without extramedullary manifestation. In their study, patients with orbital myeloid sarcoma and central nervous system myeloid sarcoma presented better outcome than patients without extramedullary manifestation, with overall survival of $92 \%$ and $73 \%$ versus $50 \%$, respectively. Patients with orbital MS 
also presented significantly lower relapse rate [39]. Similar results have been already shown in the study by Dusenbury et al., who demonstrated that non-skin extramedullary involvement was a favourable prognostic factor in pediatric patients with AML [40].

Contrary to orbital MS, cutaneous MS is associated with an aggressive course of the disease. In the analysis by Hurley et al., which included 83 adult patients with cutaneous myeloid sarcoma, the one-year mortality rate was $71.1 \%$ [23]. In other reports, leukemia cutis has also been considered as a marker of an aggressive course of the disease [3, 22, 27].

In the report by Kobayashi et al., complete remission rate was lower for pediatric patients with extramedullary involvement, however it did not influence the survival rate and event free survival. Prognosis was poor in patients with MS and WBC over $100 \times 10^{9} / /[11]$.

Prognosis for patients with isolated extramedullary relapse after alloHSCT is poor, with a two year survival rate of 11-38\% (data concerning adults) [19]. However, it has been shown in three reports, that the outcome of patients with isolated extramedullary relapse is better than in patients with systemic relapse, or both systemic and extramedullary relapse [19-21].

\section{Therapeutic options}

The therapeutic algorithm for MS is unclear in children and adults. Prospective trials are lacking, therefore treatment recommendations are based on retrospective studies which focus on adult patients. Available treatment options include systemic chemotherapy, surgical resection, radiotherapy, haematopoietic stem cell transplantation (HSCT) or a combination of these methods.

In isolated MS, it is recommended to start AML conventional chemotherapy as soon as possible, as it is known that untreated MS will almost always progress to AML [41]. Chemotherapy includes standard daunorubicin and cytarabine induction therapy, followed by high-dose cytarabine. If the response is not fully achieved, radiotherapy can be considered, as a consolidation therapy [33].

In MS associated with systemic disease, AML chemotherapy is also recommended, with consideration of alloHSCT, especially if other unfavourable prognostic factors are present (age, cytogenetics) [3, 5, 41]. The role of alloHSCT is highlighted in several studies, in which it has been shown that alloHSCT improves the overall survival of patients with MS.

Chevalier et al. showed the results of treatment of 99 adults with the diagnosis of isolated or leukemic MS, who have undergone alloHSCT. The 5-year overall survival rate was $48 \%$, with no significant difference between leukemic MS and non-leukemic MS group [42]. Other authors also agree that alloHSCT may be the most efficient element of the MS therapy $[3,14]$. In the analysis by Pileri et al., the overall survival at 48 months was $76 \%$ in patients who underwent auto- or alloHSCT, compared with $0 \%$ in patients, whose treatment did not include transplantation [14]. Avni and Koren-Michowitz also showed that alloHSCT had significant impact on the overall survival prolongation in patients with MS [5].

Radiotherapy is mainly advised as a palliative treatment, to diminish the effects of tumour compression, or pain and pruritus associated with leukemia cutis. Surgery is indicated only if specific complication such as obstruction or compression due to the localization of the tumour occurs [3, 5, 41].

In cases of the recurrence of MS, with or without bone marrow involvement, reinduction chemotherapy and alloHSCT is recommended. In case of post-transplant relapse, donor lymphocyte infusion (DLI), immunosuppression or radiotherapy can be considered.

Molecularly targeted therapy can also be therapeutic option, however knowledge about this kind of treatment is mainly limited to case reports. Ando et al. presented a case of an adult male diagnosed with AML, with disseminated extramedullary post-transplant relapse, treated with gemtuzumab ozogamicin (GO), which is an antibody against CD33. Described patient has remained in remission for at least 10 months since the end of the GO treatment [43].

Patients with diagnosed $C M L$, with $B C R-A B L$ positive myeloid sarcoma can benefit from kinase inhibitors therapy with the consideration of subsequent alloHSCT $[16,17]$.

\section{Conclusions}

Among the many unclear aspects of pediatric myeloid sarcoma, de novo subtype is the major challenge. Diagnostic difficulties in this entity are associated with its basic features. Due to various clinical manifestations depending on the localization of the tumour, patients are often hospitalized in different clinics, before they are admitted to oncology units.

Development of the final leukemic presentation which draws attention toward the appropriate diagnosis may take some time. The longest noted period between presentation of the tumour and leukemic infiltration of bone marrow is 7 years [44]. Usually, the mean time until systemic disease occurs is about 10 months [2].

Recognition of MS is more challenging, as a broad panel of diagnostic tools has to be used. The diagnostic approach must include different imaging studies, histological, immunohistochemical and genetic analysis of blood, bone marrow and if possible, tumour tissue. The best diagnostic approach to recognizing MS is a biopsy of the tumour and immunohistochemical examination. However, the standard immunohistochemical panel in diagnosing MS is lacking which makes it difficult to distinguish myeloid sarcoma from other neoplasms, in particular non-Hodgkin lymphomas.

MS also remains a therapeutic challenge, as no established treatment strategy exists.

With so much to learn, an international registry of pediatric MS as a cooperative group study is required for the acquisition of knowledge about this disorder with particular focus on isolated tumours.

\section{The authors declare no conflict of interest.}

\section{References}

1. Swierdlof SH, Campo E, Harris NL, et al. (eds.). WHO classification of tumours of heaematopoietic and lymphoid tissues. International Agency for Research on Cancer, Lyon 2008.140p.

2. Klco JM, Welch J, Nguyen T, et al. State of art in myeloid sarcoma. Int Lab Hem 2011; 33: 555-65. 
3. Bakst RL, Tallman MS, Douer D, Yahalom J. How I treat acute myeloid leukemia. Blood 2011; 118: 3785-93.

4. Bain EE, Rothman I, Lin L. De novo myeloid sarcoma in a 4-monthold infant: a case report and review of the literature. J Cutan Pathol 2013; 40: 321-5.

5. Avni B, Koren-Michowitz M. Myeloid sarcoma: current approach and therapeutic options. Ther Adv Hematol 2011; 2: 309-16.

6. Ohanian M, Faderl S, Ravandi F, Pemmaraju N, Garcia-Manero G, Cortes J, Estrov Z. Is acute myeloid leukemia a liquid tumor? Int Cancer 2012; 133: 534-44.

7. Stefanidakis M, Karjalainen K, Jaalouk DE, Gahmberg CG, O’Brien S, Pasqualini R, Arap W, Koivunen E.. Role of leukemia cell invadosome in extramedullary infiltration. Blood 2009; 114: 3008-17.

8. Feng S, Cen J, Huang Y, Shen H, Yao L, Wang Y, Chen Z. Matrix metalloproteinase- 2 and -9 secreted by leukemic cells increase the permeability of blood-brain barrier by disrupting tight junction proteins. PLoS One 2011; 6: e20599.

9. Wang C, Chen Z, Li Z. The essential roles of matrix metalloproteinase-2, membrane type 1 metalloproteinase and tissue inhibitor ofmetalloproteinase- 2 in the invasive capacity of acute monocytic leukemia SHI-1 cells. Leuk Res 2010; 34: 1083-90

10. Faaij CM, Willemze AJ, Révész T, et al. Chemokine/chemokine receptor interactions in extramedullary leukaemia of the skin in childhood AML: differential roles for CCR2, CCR5, CXCR4 and CXCR7. Pediatr Blood Cancer 2010; 55: 344-8.

11. Kobayashi R, Tawa A, Hanada R, Horibe K, Tsuchida M, Tsukimoto I; Japanese childhood AML cooperative study group. Extramedullary infiltration at diagnosis and prognosis in children with acute myelogenous leukemia. Pediatr Blood Cancer 2007; 48: 393-8.

12. Dusenbery KE, Howells WB, Arthur DC, et al. Extramedullary leukemia in children with newly diagnosed acute myeloid leukemia: a report from the Children's Cancer Group. J Pediatr Hematol Oncol 2003; 25: 760-8.

13. Johnston DL, Alonzo TA, Gerbing RB, Lange BJ, Woods WG. Superio outcome of pediatric acute myeloid leukemia patients with orbital and CNS myeloid sarcoma: a report from the Children's Oncology Group. Pediatr Blood Cancer 2012; 58: 519-24.

14. Pileri SA, Ascani S, Cox MC, et al. Myeloid sarcoma: clinico-pathologic, phenotypic and cytogenetic analysis of 92 adult patients. Leukemia 2007; 21: 340-50.

15. Rénard C, Girard S, Pracros JP, Dijoud F, André JM, Mialou V, Bertrand Y. Granulocytic sarcoma, a diagnostic challenge: 3 pediatric cases. Arch Pediatr 2010; 17: 149-53.

16. Kuan JW, Pathmanathan R, Chanq KM, Sen-Moi Tan. Aleukemic bcrabl positive granulocytic sarcoma. Leuk Res 2009; 33: 1574-7.

17. Mitchell M, Itani D, Gerber J, Ghosh N, Gojo I, Zeidan A. Thoracic $t(9 ; 22)$ positive granuloxytic sarcoma as initial presentation of chronic myeloid leukemia. Clin Lymphoma Myeloma Leuk 2013; 13: 619-21.

18. Yoo SW, Chung EJ, Kim SY, Ko JH, Baek HS, Lee HJ. Multiple extramedullary relapses without bone marrow involvement after second allogeneic hematopoietic stem cell transplantation for acute myeloid leukemia. Pediatr Transplant 2012; 16: 125-9.

19. Harris AC, Kitko CL, Couriel DR, et al. Extramedullary relapse of acute myeloid leukemia following allogeneic hematopoietic stem cell transplantation: incidence, risk factors and outcomes. Haematologica 2013; 98: 179-84

20. Solh M, DeFor TE, Weisdorf DJ, Kaufman DS. Extramedullary relapse of acute myelogenous leukemia after hematopoietic stem cell transplantation: better prognosis than systemic relapse. Biol Blood Marrow Transplant 2012; 18: 106-12.

21. Clark WB, Strickland SA, Barrett AJ, Savani BN. Extramedullary relapses after allogeneic stem cell transplantation for acute myeloid leukemia and myelodysplastic syndrome. Haematologica 2010; 95: 860-3.

22. Cho-Vega JH, Medeiros LJ, Prieto VG, Vega F. Leukemia cutis. Am J Clin Pathol 2008; 129: 130-42.

23. Hurley MY, Ghahramani GK, Frisch S, et al. Cutaneous myeloid sarcoma: natural history and biology of an uncommon manifestation of acute myeloid leukemia. Acta Derm Venereol 2013; 93: 319-24.

24. Choi JH, Lee HB, Park CW, Lee CH. A case of congenital leukemia cutis. Ann Dermatol 2009. 21: 66-70.
25. Uihlein LC, Brandling-Bennett HA, Lio PA, Liang MG. Sweet syndrome in children. Pediatr Dermatol 2012; 29: 38-44.

26. Shvartsbeyn M, Pandey S, Mercer SE, Goldenberg G. Leukemia cutis presenting clinically as disseminated herpes zoster in a patient with unrecognized acute promyelocytic leukemia. J Clin Aesthet Dermatol 2012; 5: 40-3.

27. Rao AG, Danturty I. Leukemia Cutis. Indian J Dermatol 2012; 57: 504.

28. Murthy R, Vemuganti GK, Honavar SG, Naik M, Reddy V. Extramedul lary leukemia in children presenting with proptosis. J Hematol Oncol 2009; 24: 2-4.

29. Roby BB, Drehner D, Sidman JD. Granulocytic sarcoma of pediatric head and neck: an institutional experience. Int J Pediatr Otorhinolaryngol 2013; 77: 1364-6.

30. Noh BW, Park SH, Chun JE, Kim JH, Kim HJ, Lim MK. Granulocytic sarcoma in the head and neck: CT and MR imaging findings. Clin Exp Otorhinolaryngol 2009; 2: 66-71.

31. Yilmaz AF, Saydam G, Sahin F, Baran Y. Granulocytic sarcoma: a systematic review. Am J Blood Res 2013; 3: 265-70.

32. Stolzel, Röllig C, Radke J, et al. F-FDG-PET/CT for detection of extramedullary acute myeloid leukemia. Haematologica 2011; 96: 1552-6.

33. Alexiev BA, Wang W, Ning Y, Chumsri S, Gojo I, Rodgers WH, Stass SA, Zhao XF. Myeloid sarcomas: a histologic, immunohistochemical, and cytogenetic study. Diagn Pathol 2007; 31: 42.

34. Campidelli C, Agostinelli C, Stitson. Myeloid sarcoma: extramedullary manifestation of myeloid disorders. Am J Clin Pathol 2009; 132 426-37.

35. Kurata H, Okukubo M, Fukuda E. Myeloid sarcoma should be undertaken in cases of CD56 positivity to exclude granulocytic sarcoma. Br J Dermatol 2002; 147: 609-11.

36. Zhang XH, Zhang R, Li Y. Granulocytic sarcoma of abdomen in acute myeloid leukemia with inv(16) and $t(6 ; 17)$ abnormal chromosome: case report and review of literature. Leuk Res 2010; 34: 958-61.

37. Falini B, Lenze D, Hasserijan R. Cytoplasmic mutated nucleophosmin (NPM) defines the molecular status of a significant fraction of myeloid sarcomas. Leukemia 2007; 21: 1566-70.

38. Tsimberidou AM, Kantarjian HM, Wen S. Myeloid sarcoma is associated with superior event-free survival and overall survival compared with acute myeloid leukemia. Cancer 2008; 113: 1370-8.

39. Johnston DL, Alonzo TA, Gerbing RB, Lange BJ, Woods WG. Superior Outcome of Pediatric Acute Myeloid Leukemia Patients with Orbital and CNS Myeloid Sarcoma: A Report from the Children's Oncology Group. Pediatr Blood Cancer 2012; 58: 519-24

40. Dusenbery KE, Howells WB, Arthur DC. Extramedullary leukemia in children with newly diagnosed acute myeloid leukemia: a report from the Children's Cancer Group. J Pediatr Hematol Oncol 2003; 25: $760-8$.

41. Lan TY, Lin DT, Tien HF, Yang RS, Chen ChY, Wu K. Prognostic factors of treatment outcomes in patients with granulocytic sarcoma. Acta Haematol 2009; 122: 238-46.

42. Chevallier P, Labopin M, Cornelissen J, Socié G, Rocha V, Mohty M, on behalf of the ALWP of EBMT. Allogeneic hematopoietic stem cell transplantation for isolated and leukemic myeloid sarcoma in adults: a report from the Acute Leukemia Working Party of the European group for Blood and Marrow Transplantation. Haematologica 2011; 96: 1391-4.

43. Ando T, Mitani N, Matsunaga K. Gemtuzumab ozogamicin therapy for isolated extramedullary AML relapse after allogeneic hematopoietic stem-cell transplantation. Tohoku J Exp Med 2010; 220: 121-6.

44. Lengerke C, Wirths S, Kanz L, Hebart H, Kaiserling E, Krober SM. Unusual leukemia presentations. Case 1. Pulmonary chloroma preceded by leukemia cutis 7 years earlier. J Clin Oncol 2005; 23: 5837-9.

\section{Address for correspondence}

\section{Katarzyna Derwich}

Department of Pediatric Oncology, Hematology and Transplantology Poznan University of Medical Sciences

Poznan, Poland

e-mail: kderwich@poczta.onet.pl

Submitted: 6.01 .2015

Accepted: 24.03 .2015 